\title{
NGS2: a focal plane array upgrade for the GeMS multiple tip-tilt wavefront sensor
}

François Rigaut, lan Price, Céline d'Orgeville, Francis Bennet, Nick Herrald, et al.

François Rigaut, Ian Price, Céline d'Orgeville, Francis Bennet, Nick Herrald, Nicolas Paulin, Kristina Uhlendorf, Vincent Garrel, Gaetano Sivo, Vanessa Montes, Chad Trujillo, "NGS2: a focal plane array upgrade for the GeMS multiple tip-tilt wavefront sensor," Proc. SPIE 9909, Adaptive Optics Systems V, 99091X (26 July 2016); doi: 10.1117/12.2230481

SPIE Event: SPIE Astronomical Telescopes + Instrumentation, 2016, Edinburgh, United Kingdom 


\title{
NGS2: a focal plane array upgrade for the GeMS multiple tip-tilt wavefront sensor
}

\author{
François Rigaut ${ }^{a}$, Ian Price ${ }^{a}$, Céline d'Orgeville ${ }^{a}$, Francis Bennet ${ }^{a}$, \\ Nick Herrald ${ }^{a}$, Nicolas Paulin ${ }^{a}$, Kristina Uhlendorf ${ }^{b}$, \\ Vincent Garrel ${ }^{c}$, Gaetano $\mathrm{Sivo}^{c}$, Vanessa Montes ${ }^{c}$, and Chad Trujillo ${ }^{c}$ \\ ${ }^{a}$ Advanced Instrumentation \& Technology Centre, \\ Research School of Astronomy and Astrophysics, \\ Australian National University, Canberra, ACT 2611, Australia ; \\ ${ }^{b}$ Jabil Optics Germany GmbH, Carl-Zeiss-Promenade 10, 07745 Jena, Germany; \\ ${ }^{c}$ Gemini Observatory, c/o AURA, Casilla 603, La Serena, Chile
}

\begin{abstract}
NGS2 is an upgrade for the multi-natural guide star tip-tilt \& plate scale wavefront sensor for GeMS (Gemini Multi-Conjugate Adaptive Optics system). It uses a single Nüvü HNü-512 Electron-Multiplied CCD array that spans the entire GeMS wavefront sensor focal plane. Multiple small regions-of-interest are used to enable frame rates up to $800 \mathrm{~Hz}$. This set up will improve the optical throughput with respect to the current wavefront sensor, as well as streamline acquisition and allow for distortion compensation.
\end{abstract}

Keywords: Adaptive Optics, Wavefront Sensing, Multi-Conjugate, Natural Guide Star, Focal Plane Sensing, Tip-Tilt Wavefront Sensor, Detectors, EMCCDs

\section{INTRODUCTION}

GeMS, ${ }^{1,2}$ the Gemini south telescope Multi-Conjugate Adaptive Optics (MCAO) system, uses five laser guide stars for high-order wavefront sensing, and up to three natural guide stars (NGS) for tip-tilt and plate scale modes sensing. The original Natural Guide Star Wavefront Sensor (NGS WFS, sometimes called TTWFS, as it is used for Tip-Tilt sensing) for GeMS is based on a rather complex, difficult to align optical setup (see section 2), and suffers from very low throughput, thereby reducing the limiting magnitude by over 2.5 , limiting the fraction of sky accessible to GeMS to $<5 \%$ rather than the design target of $30 \%^{*}$.

An additional serious limitation is linked to field distortion: The GeMS optical bench, CANOPUS, uses a F/16-F/16 two-parabola optical design to feed the WFSs. This introduces significant focal plane geometrical distortion. As a result of these distortions, a star asterism will effectively change shape when it is offset across the output focal plane. Because the guide probes (see section 2) do not follow these distortions, the current NGSWFS can not offset by more than typically $10^{\prime \prime}$ without necessitating a re-acquisition of the star asterism.

In 2014, the Australian National University (ANU) secured funding from the Australian Research Council (ARC), ANU and other partners (Gemini, the Australian Astronomical Observatory and the Swinburne University of Technology) to design and build NGS2 ${ }^{\dagger}$ to replace GeMS's current NGSWFS. This new WFS is based on a single Electron-Multiplied $\mathrm{CCD}^{3}$ (EMCCD) focal plane array and uses multi regions-of-interest to read at rates up to $800 \mathrm{~Hz}$, with a read out noise of typically $0.3 \mathrm{e}^{-}$. It will provide a sensitivity gain of more than 2.5 magnitudes, and will vastly improve or solve operational issues related to acquisition and offsetting. NGS2 is being integrated at ANU, and will undergo factory acceptance testing at ANU in 2016 before commissioning at Gemini South in 2017.

Further author information: (Send correspondence to Francois Rigaut)

Francois Rigaut: E-mail: francois.rigaut@anu.edu.au, Telephone: +61 261250210

* Sky coverage is of course more complicated than just a single scalar number, especially for GeMS. It depends on the galactic latitude, the number of guide stars $(1,2$ or 3$)$ and the criteria used for Strehl degradation. These numbers are just indicative and are given for 3 guide star constellations at 60 degrees galactic latitude.

${ }^{\dagger}$ Natural Guide Star Next Generation Sensor $=$ NGSNGS $=$ NGS $^{2}$ or NGS2 for convenience and plain text compatibility 


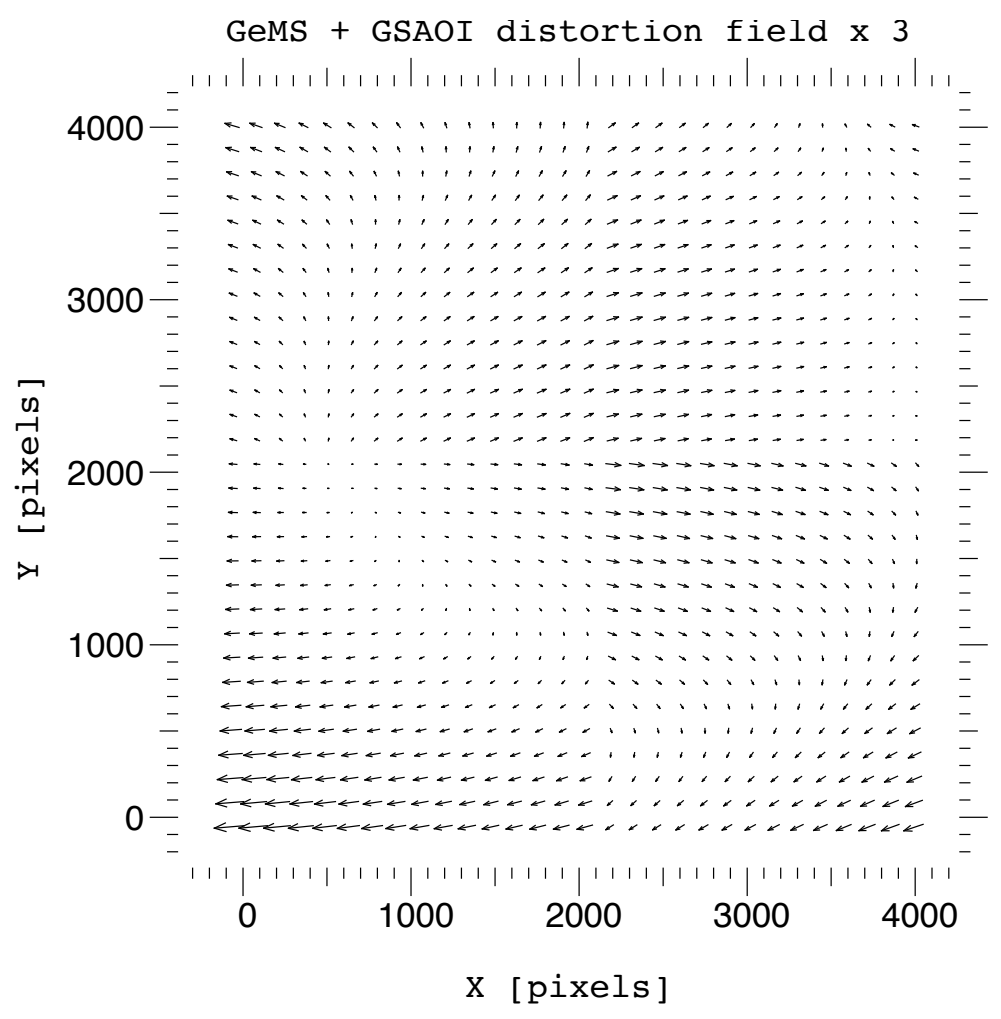

Figure 1. CANOPUS science focal plane field distortions. One pixel is approximately 20 mas. Distortions of $2^{\prime \prime}$ are common throughout the field. Distortions in the lower left corner reach $4^{\prime \prime}$. Note that translation and overall scaling have been removed. This map was obtained by calibration on a known astrometric field. ${ }^{4}$

\section{GEMS ORIGINAL NGS WFS}

The current NGS WFS suffers from a number of issues: very low optical throughput, and uncorrected/unmodeled focal plane distortions that impact acquisition time and the ability to offset, all issues that are detailed below.

The current GeMS Tip-Tilt multi-NGS WFS uses Avalanche Photo-Diodes (APD). APDs are well suited to the low counts generally dealt with when using faint TT guide stars. APDs are photon counting devices with zero read-out noise and, for the APDs used in CANOPUS, typically 400 counts of dark current per second. Unfortunately they are bulky and difficult to integrate into optics. The current NGSWFS is using a rather complex optical design, with pyramids ${ }^{\ddagger}$ in the focal plane feeding the light to fibres that transmit it to the APD themselves. Optical components are tiny, and very difficult to align. Multiple genuine attempts have already been made to fix or improve this system, with little success; The current optical throughput is estimated to be around $4 \%$, drastically reducing the sensitivity of the NGSWFS and directly impacting GeMS sky coverage to around $5 \%$ instead of the $30 \%$ initially specified due to the Tip-Tilt NGS sensitivity.

Guide star acquisition is done by moving the guide probe in the field: Each of the three APD-based TT probe/sensor has a field of view diameter of $1.4^{\prime \prime}$ and is mounted on a XY stage. Probes $1 \& 2$ are physically mounted on probe 3 . The probe 3 light is split between a TT-APD WFS and a focus sensor, used to monitor slow focus drift due to the sodium layer altitude changes. Probe 3 also adds a $\mathrm{Z}$ stage, which allows to optimise image sharpness on the guide probe. Unfortunately, partly due to the fact that the TTWFSs are quad-cells, and the direct consequence on the instrument focusing through the probe 3 focus sensor and focus loop, the optimisation of the guide probe Z-position is cumbersome and rarely done.

\footnotetext{
${ }^{\ddagger}$ Note that this has nothing to do with a pyramid sensor, as each facet of the pyramid feeds a single detecting element. In effect, this is a simple quad-cell.
} 
The CANOPUS optical design is based on a $\mathrm{F} / 16$ input parabola ${ }^{\S}$, producing a collimated beam in which the multiple deformable mirrors are placed. A dichroic sends the light short of $850 \mathrm{~nm}$ toward the WFS assembly, while the light above $850 \mathrm{~nm}$ is sent to the science instrument. In the WFS path, another F/16 parabola reforms a focus at which the NGS TT WFS is placed. In the science field, a F/32 parabola is used, which produces a plate scale suitable for use with GSAOI, or any other instrument. It is well know that this two-parabola relay arrangement produces significant focal plane distortions. Figure 1 shows the distortion field in the science focal plane, calibrated on-sky. ${ }^{4}$ The equivalent distortion in the WFS focal plane has never been calibrated, and this calibration would be extremely challenging, or simply impossible to realise with the current hardware. The consequence of these distortions is that the three-NGS asterism will effectively change shape when it is offset across the field of view. Because the guide probes move rigidly (only probe 3 is moved and drags $1 \& 2$ in the process, see above), the probe positions do not match the NGS positions after the offset. Through the plate scale loop and the offload of the rotation term to the Cassegrain rotator, these position errors will change the plate scale and rotation in the science focal plane, making it more difficult to stack them during the data reduction process. Note that the effect on the science image is just the two plate scale modes and rotation, so is only really an issue for very empty science fields (otherwise stars \& objects can be used to fit a simple scale/rotation model). A more serious issue arises for larger telescope offsets (typically $>10^{\prime \prime}$ ), for which this probe/NGS mismatch becomes large enough that the star may not be in the probe acquisition field anymore, necessitating a re-acquisition, thus breaking the automation of the acquisition sequence. This is a significant operational and performance limitation that needs solving.

Another consequence of the distortions is that initial guide star acquisition is sometimes a problem. The distortion can be arcseconds, and thus sometimes the star is off the APD acquisition field, especially because there is no distortion model in the probe positioning model. This sometime leads to lengthy acquisition time.

\subsection{A technological opportunity}

In recent years, new focal plane devices have become available that offer simultaneously low noise, speed and a large number of pixels. This makes it possible to use such a detector to cover the entire GeMS field of view, and pick and use multiple guide stars therein. There are multiple advantages in doing so, compared to the current NGSWFS implementation, which are listed below:

- Direct imaging of the entire focal plane on an array makes for a more direct optical feed, requiring less exotic optics, and thus necessarily resulting in better optical throughput;

- Acquisition procedures are significantly simplified and streamlined. Currently, finding guide stars can be a very lengthy procedure, involving lengthy spiral searches. The associated overhead can be up to 10min, sometimes larger. The focal plane array will provide the entire field of view in one single snapshot, and will allow the operator to quickly pick up the brightest guide stars;

- There is no vignetting by the other probe arms, thus cancelling the need for guide probe patrol area limitations;

- The number of mechanisms is greatly reduced, as there is no need for guide probe and thus no moving part for the essential guide star acquisition functions, greatly improving robustness and longevity. The only mechanism is the focus stage, see below (this adds a functionality that did not exist in the current GeMS TTWFS and will result in further SNR gain by insuring that the image is always in focus on the detector, see next item);

- The use of a focal plane array instead of a simple quad-cell allows to measure the spot size, and to optimise it with the focus stage (the current version has neither the ability to measure nor to correct focus error);

- It is easier to tailor the field of view (FoV) to the object with region-of-interest than an aperture in front of the APD: Smaller FoV in good seeing, larger FoV for extended objects, etc, procuring potential gain in SNR and the ability to use extended bright objects;

\footnotetext{
$\S$ This refers to the F-ratio at the output of the parabola, assuming a collimated beam input - or vice versa.
} 


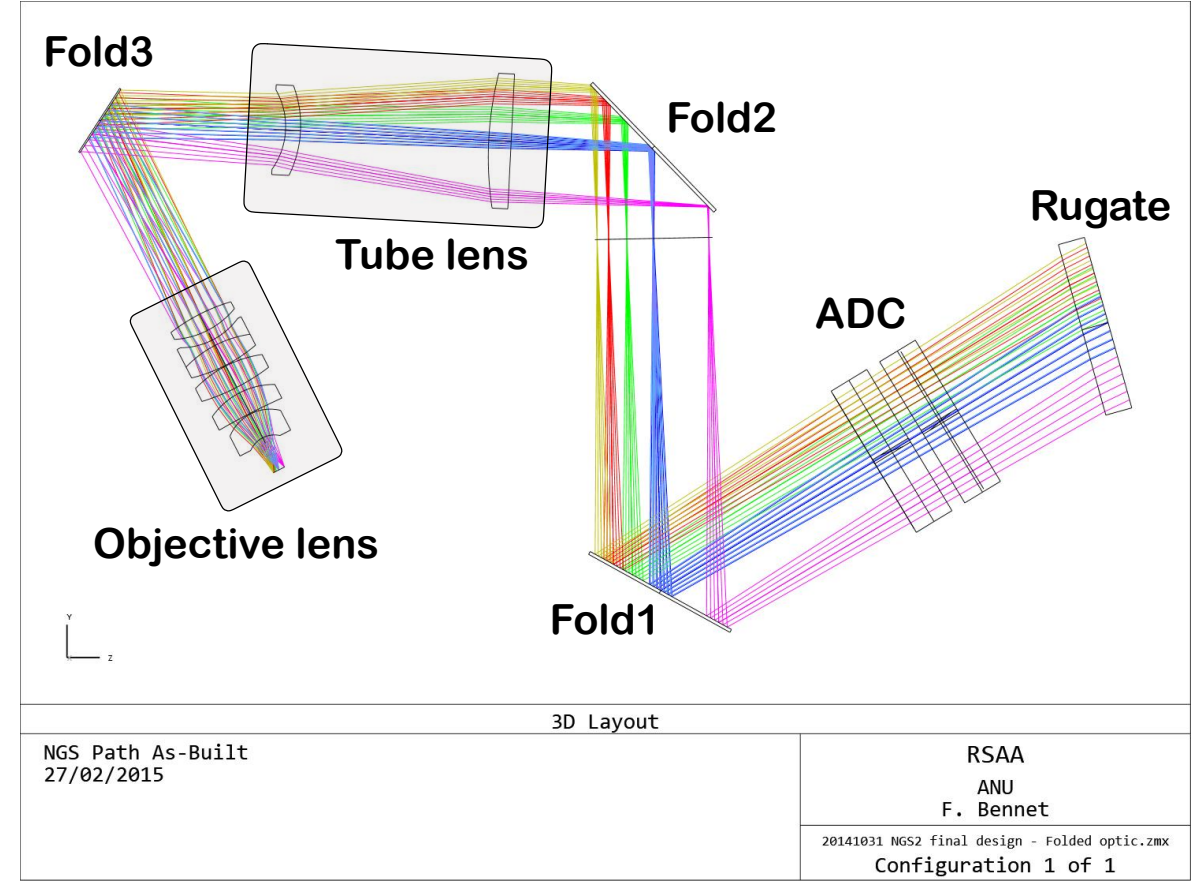

Figure 2. NGS2 optical layout. The input is at F/16, the output at F/1.80. A calibration unit can conveniently be placed at the focal plane just upstream of Fold 2.

- Most importantly, NGS2 compensates for distortions, i.e. the guide window positions take into account the NGSWFS field distortion (calibrated on sky during commissioning), so that the guide window follows and compensates for the distortion of the guide star asterism.

This new TTWFS concept does not come without a few limitations:

- There is no way to do the focus sensing to compensate for sodium layer altitude variations, as is done currently by probe 3 (see section 5.1 for a discussion on how this is dealt with),

- The choice of a single array imposes some limitations on the difference of brightness between the three guide stars (see section 6): the brightest of the guide stars limits the maximum Electron Multiplied (EM) gain that can be used, and might not be optimal for the fainter stars also used in the field.

Far and away advantages outweigh limitations. As far as cost, there is no huge difference between the two sensor types (APD-pyramid and EMCCD).

Detectors to choose from include sCMOSs and EMCCDs. Their respective merits are discussed in Sec 4.1.

\section{NGS2 REQUIREMENTS}

NGS2 has been designed based on a number of requirements that have been established by Gemini and the ANU. The most relevant requirements have been consolidated and summarised below:

- NGS2 shall do Tip-Tilt sensing on up to 3 GS, anywhere in the CANOPUS field of view (no limitation in position);

- NGS2 shall use a focal plane array;

- NGS2 shall reach frame rates of up to $800 \mathrm{~Hz}$ (to match LGS loop);

- NGS2 optical design shall result in no vignetting and ghosts lower than $10^{-4}$ of the primary image;

- NGS2 measurement chain shall result in less than 35mas rms noise on a magnitude $\mathrm{R}=17$ star (assuming $185 \mathrm{ph} /$ frame at $200 \mathrm{~Hz}$ in the $\mathrm{R}$ band at the input of NGS2 for a $\mathrm{R}=17 \mathrm{star}$ ); 
- NGS2 shall provide a focus adjustment to minimise image size and maximise SNR;

- NGS2 implementation should have the least impact on GeMS (opto-mechanical, software, electronics, enclosures and operations);

- No heat and no light shall be released in the CANOPUS enclosure.

\section{DESIGN}

In this section, we present the NGS2 detector trade studies, and the opto-mechanical and software design.

\subsection{Detector}

We initially investigated the use of sCMOS detectors. These now offer sub-electron read-out noise (median value), large arrays, and relatively fast frame rates. However, sCMOS were not totally mature at the time of detector selection, and there were concerns about the non-uniformity of noise and the latency, following informal reports of initial tests in the AO community (which turned out since then to be mostly unfounded). Another issue with these detectors is the small pixel size, typically 6.5 microns, which would have made the optical design challenging, requiring an end F-ratio of $\approx 0.75$.

We then turned to EMCCD and found that Nüvü had initial plans to develop a multi regions of interest (mROI) mode for their HNü-512 EMCCD for different applications. mROI mode is somewhat common in low end camera but is generally not offered in high end low noise EMCCDs. Nüvü embarked into the development and by mid 2015 delivered a HNü-512 including mROI capability. Test of the detector are reported in section 6.1.

\subsection{Optical}

The optical layout is shown on figure 2. The Rugate filter and the atmospheric dispersion compensator (ADC) are pre-existing elements. The Rugate reflects the 589-nm light toward the high order LGSWFS (Laser Guide Star WFS) and lets all other wavelengths into the NGSWFS. There is an intermediate focal plane just before Fold 2, which is necessary and convenient to calibrate NGS2. The tube lens is made of two lenses, and the objective lens of 5 lenses (one is a doublet). The objective lens output is at F/1.84. To make it fit within the allowable space envelope, the beam has to be folded at three locations.

The specifications and design results are listed in table 1. The optical quality is exceeding the requirements (see table 1 and figure 3), as well as the optical throughput. The ghost analysis reveals a couple of ghosts with contrasts of 1:85000 (12.3 magnitudes, only visible for FoV $\left.<0.1^{\prime}\right)$ and 1:2 106 (15.7 magnitude).

Table 1. NGS2 optical subsystem specifications

$\begin{array}{lll}\text { Parameter } & \text { Specification } & \text { As designed } \\ \text { Detector } & \text { HNü-512 EMCCD } & \\ \text { Format } & 512 \times 512 & \\ \text { Pixel size } & 16.0 \mu \mathrm{m} & \\ \text { Field of View } & 120^{\prime \prime} \times 120^{\prime \prime} & 119^{\prime \prime} \times 119^{\prime \prime} \\ \text { Pixel scale } & 0.235^{\prime \prime} / \text { pixel } & 0.233^{\prime \prime} / \text { pixel } \\ \text { F-number } & 1.84 & 1.80 \\ \text { Wavelength range } & 450-565 \mathrm{~nm} ; 595-950 \mathrm{~nm} & 450-950 \mathrm{~nm} \\ \text { Aberrations } & >80 \% \text { of rays in pixel } & >95 \% \\ \text { Distortion } & <5 \% & <2 \% \\ \text { Telecentricity } & \text { No requirement } & <3.5^{\circ} \\ \text { Interface } & \text { F } / 16 \text { as current CANOPUS NGSWFS } & \\ \text { Overall volume } & \text { Has to fit in available NGS WFS space } & \\ \text { Overall Transmission } 500-800 \mathrm{~nm} & >70 \% & >87 \% \\ \text { Overall Transmission } 450-950 \mathrm{~nm} & >70 \% & >81 \%\end{array}$




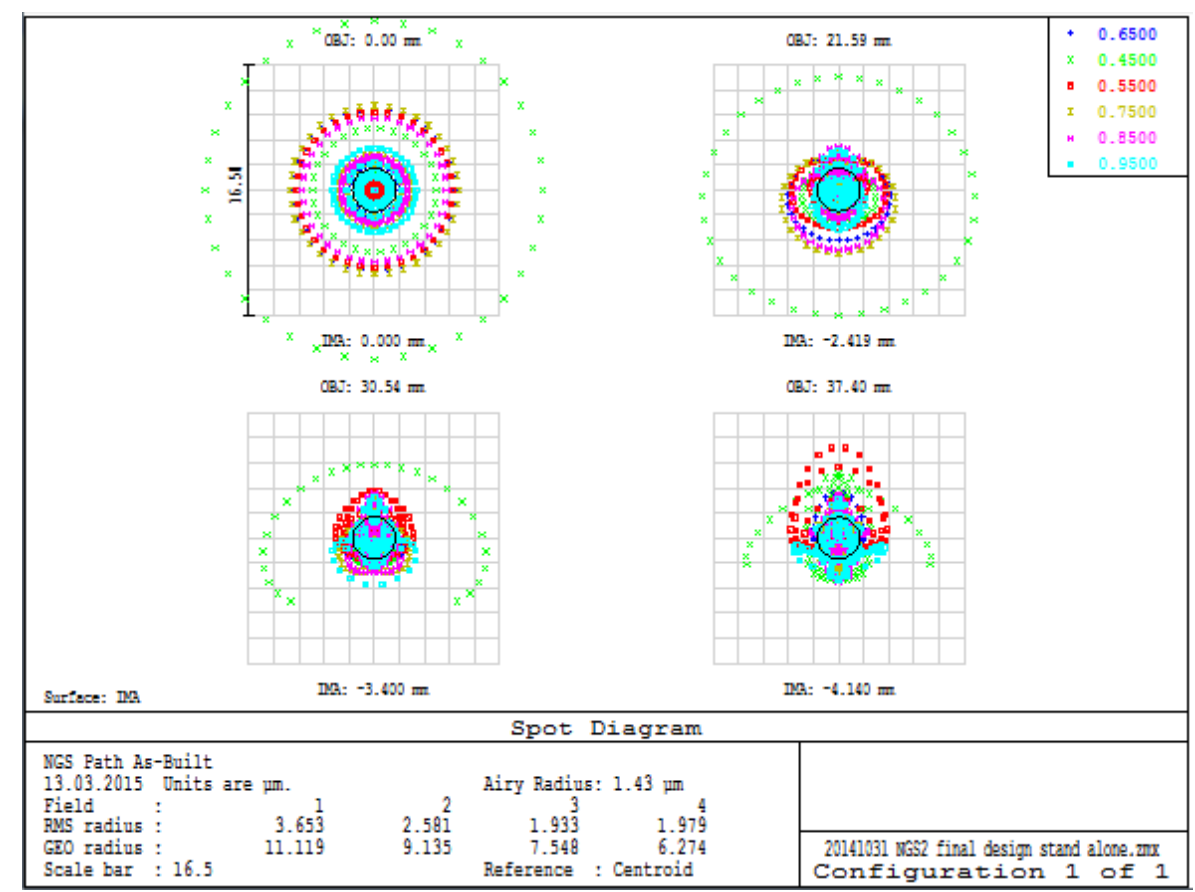

Figure 3. NGS2 spot diagram. Each full grid is a $16 \mu \mathrm{m}$ pixel. The upper left spot diagram is for an on-axis source. The other spot diagrams are for objects at various off-axis distances, as indicated on the figure $\left(1^{\prime \prime}\right.$ is $621 \mu \mathrm{m}$ at the F/16 NG2 input focal plane).

The optical adjustment concept involves adjusting the position of lenses in the objective lens assembly. Spherical aberration, field coma and on-axis coma can be minimised by changing the distance between and the lateral position of some identified lenses. This should however not be required, as mechanical tubes have been manufactured with the required, tight tolerances (see section 4.3).

\subsection{Mechanical}

\subsubsection{Fold Mirrors}

The three flat fold mirrors are kinematically first surface mounted, between two fixed balls and a third ball end, fine adjustment screw (254 tpi), which controls the pitch. Yaw adjustment is achieved by rotation of the housing about a pin also using fine adjustment screws.

The two large folds at the beam input to the sensor form a periscope which gives the freedom to control the location and angle of the optical axis at the first lens in the sensor, by adjustment of the pitch and yaw of the two folds. This is important in that it allows the sensor to be aligned with the rest of the instrument, despite the lack of definition of the focus location within Canopus.

\subsubsection{Objective Assembly}

The objective assembly consists of a set of lenses, stacked within a common bore. The lenses are separated by spacers which make toroidal contact with the lens surfaces. The stack is pre-loaded by a single wave spring. The objective lens housing is nested semi-kinematically within a V-Groove, and located in focus by a dowel pin, allowing the objective assembly to be removed and replaced repeatably if required.

\subsubsection{Design Challenges}

The decentre tolerances for the optics in the objective are very tight. Centring of the optics is provided either by the fit between the mechanical diameters on the lenses and the bore of the 304 stainless steel lens housing, or by fine threaded adjustment screws in the lens housings which are able to push the lenses laterally. The tightness of 


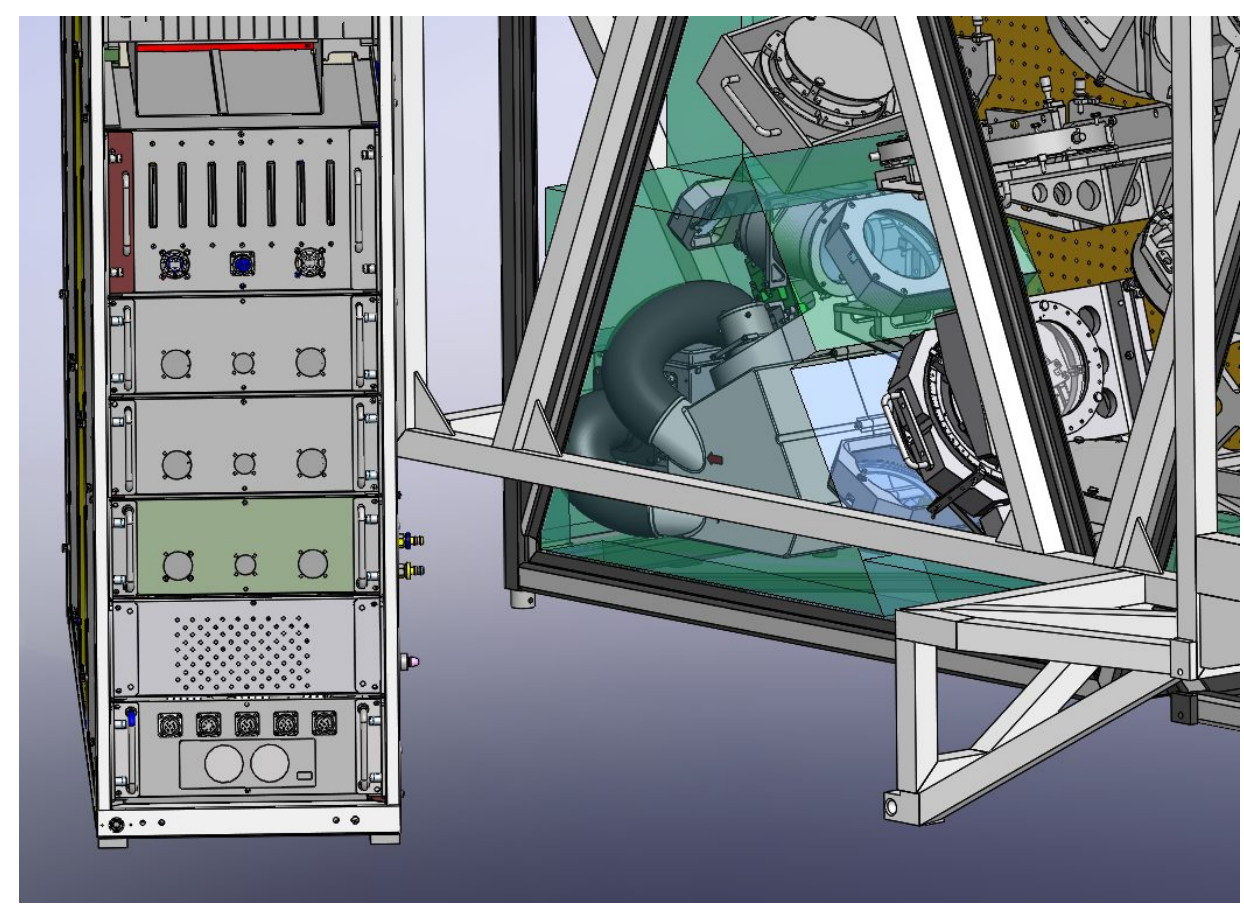

Figure 4. NGS2 as it fits in CANOPUS, occupying the volume of the current NGS WFS. On the left, the thermal enclosure containing the electronic cabinet, in which the NGS2 electronics will be located.

the diametral fit is constrained by the differences in thermal strain between the various glasses of the objective and the steel housing, over the relatively wide survival temperature range of -15 to 25 degrees C. Four of the six lenses in the objective are made from $\mathrm{CaF} 2$, which has a much higher coefficient of thermal expansion than the other glasses, and one which is slightly higher than 304 stainless.

To accommodate the tight optical tolerances, the lens housing bore is sized such that there is always clearance between the CaF2 lens and housing diameters over the entire survival temperature range. The additional diametral clearance required by the two non-CaF2 lenses is taken up by a pair of novel, conforming, centring spacers. These spacers incorporate two sets of overlapping circumferential grooves, which act to distribute the thermal strain over the thin inner section of the spacer, whilst maintaining centring of the optic.

The lens spacers are designed to make tangential contact with the lens surfaces, in order to maximise mounting accuracy. This results in Hertzian contact stresses in the lenses. This is compounded by the use of CaF2 lenses, which are extremely fragile. To minimise the stresses in the CaF2 lenses, the contacting spacer faces were made toroidal, such that the spacer face cupped the spherical lens surface at a slightly larger diameter than the lens itself. The toroidal surfaces were required to be highly accurate to ensure that there were no unexpected centres of stress concentration due to manufacturing errors. Significant effort was expended in both the manufacture and the verification of the parts through CMM measurements to ensure that the toroidal surfaces were made to tolerance.

\subsubsection{Camera Mount and Thermal}

The Nüvü camera is large and heavy. The camera bracket which provides the mounting was made very stiff to ensure that flexure due to a changing gravity vector does not compromise the overall system alignment. Figure 5 shows the mounting bracket, the objective lens located on the focus stage, and the HNü-512 camera.

Cooling of the Nüvü camera detector and on-board amplifiers is achieved through both fluid cooling and fan driven, airflow based cooling. A requirement was that no heat be introduced to the Canopus instrument. An enclosure was designed and produced through $3 \mathrm{D}$ printing, to contain the warm air exhausted by the camera. 


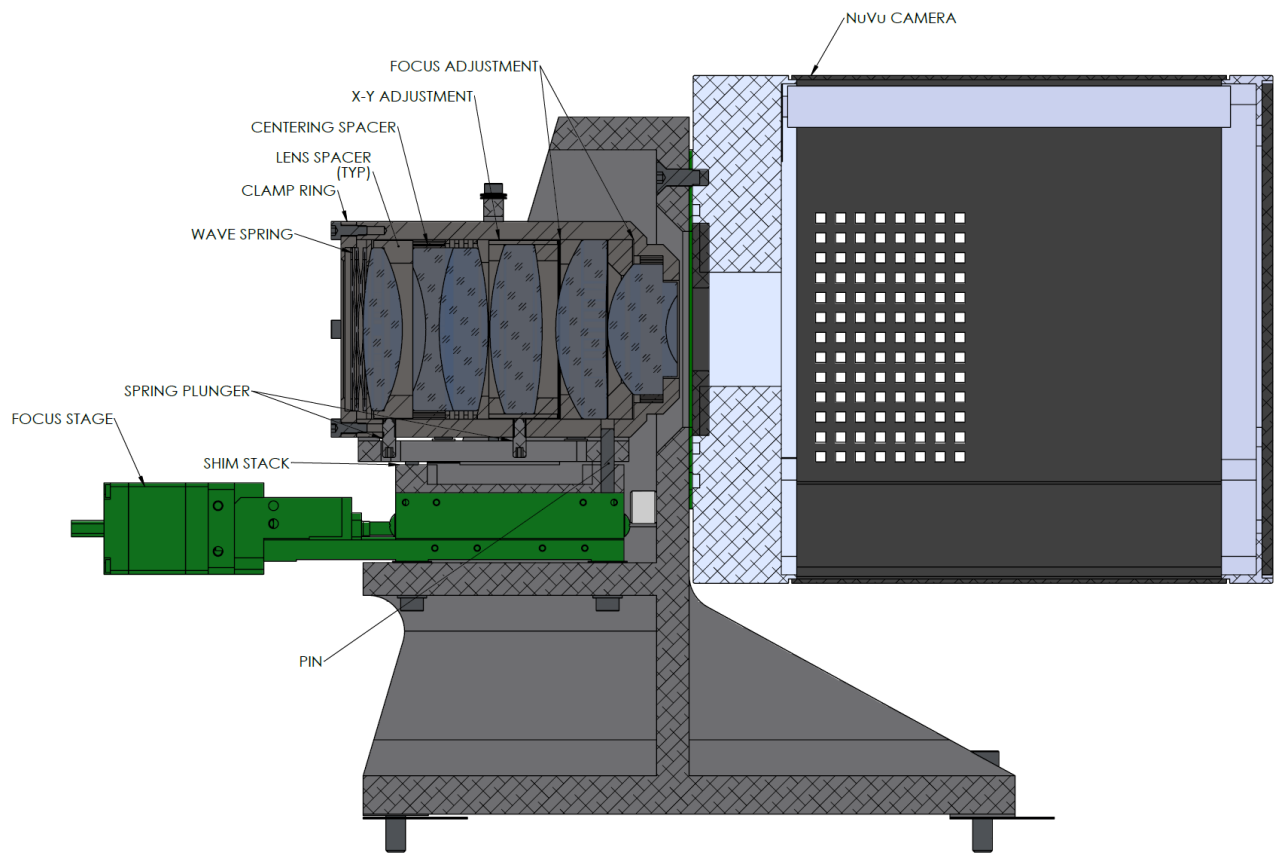

Figure 5. Objective lens and focus stage. The fine adjustment focus stage moves the objective assembly with respect to the detector. It allows coarse focusing (after e.g. a change of instrument) and fine focusing (to maximise the NGS2 spot position measurement SNR). It also compensates for thermally induced defocus.

The contained air will be driven through an air-liquid heat exchanger to be located adjacent to the Canopus Optical Bench. The fluid cooling will be taken care of by the Gemini facility chilled water systems.

\subsubsection{Split Baseplates}

The sensor components are mounted to a pair of common base plates. Originally it was intended that the entire system be mounted to a single base plate, however this would have proved impractical at integration due to the access restrictions imposed by the CANOPUS frame and $(+\mathrm{X})$ Thermal Enclosure (see figure 4$)$. The base plate was split such that the input periscope forms one assembly, and the rest of the optics plus the detector forms the second assembly. The splitting of the base plate simplifies the integration procedure enormously, without significant costs in terms of subsequent alignments. The two base plates incorporate kinematic registration elements, meaning that they can be repeatably positioned. Also, the alignment functions provided by the periscope allows greater freedom in the positioning of both base plates relative to the input beam from Canopus.

\subsection{Software}

The NGS2 control system was required to be a drop-in replacement of the existing system to the greatest extent possible. To this end, the NGS2 control software emulates the three robotic arm positioners, mapping the commands to position the linear actuators to a multiple region-of-interest (mROI) configuration of the detector system and guide star reference positions in pixel space. The robotic positioning was implemented in EPICS and the NGS2 software provides a soft IOC (Input/Output Communication) that matches the recordlevel interface of the current system. Control of the detector system, data acquisition and processing of the pixel data is implemented in a separate process. This process runs on the same host as the soft IOC and is completely independent of EPICS. The ZeroMQ (0MQ) middleware is used for Inter-Process Communication (IPC) between the detector control process and the soft IOC. It is also used for control and monitoring via an engineering Graphical User Interface (GUI).

The original NGSWFS system used the Gemini implementation of the EPICS Coordinated Motion Record for each robotic positioner, utilising the $\mathrm{X}$ and $\mathrm{Y}$ axes of each of the three record instances. The NGS2 software 
replaces the Coordinated Motion Records for the positioners with Virtual Probe records. These records have precisely the same set of fields, though many fewer are relevant. The seemingly independent Virtual Probe records are collated at the EPICS device layer, since they are all associated with a single hardware device. Other EPICS records provide configuration inputs and telemetry outputs. Inputs specify things such as the operating mode (full or windowed), the window size for each guide star and the number of frames to co-add before processing the pixels to a source position. Outputs include the measured guide star positions and flux. Many of these records are new to the system, since they were not applicable to the APD-based design, but the EPICS interface enables integration with the MCAO system with relatively few changes. In all cases, the device layer of the NGS2 EPICS records communicate via $0 \mathrm{MQ}$ with a server that is part of the detector control process.

The detector control process manages the device configuration and control, the focus motor control, pixel data acquisition, telemetry, pixel processing and communication of the measured guide star position errors to the MCAO real time controller (RTC). Commands from the engineering GUI or NGS2 soft IOC that relate to the window size or guide star location are delegated to a reconfiguration task. This task determines whether the current detector readout mode and mROI configuration is appropriate, computing a new configuration if needed. Guide star locations in the focal plane are mapped to pixel positions via a transformation that include a model for field distortion. This provides the reference for position measurements. It is rounded to the nearest integer to compute a window center. If the windows are not encapsulated by the current mROI configuration a new $\mathrm{mROI}$ is computed and issued to the detector system. In determining the new mROI the requested window sizes are increased by adding a one pixel border. This margin reduces the occurrence of reconfiguration of the mROI for sub-pixel changes in the input guide star location that result from flexure and differential atmospheric dispersion corrections. The set of windows are constrained to the full array boundaries and merged into one to three region-of-interest bands based on the row range of the windows.

Data acquisition pixel processing and communication with the RTC are sequential operations in the data processing task. This task generally operates asynchronously from other tasks in the detector control process, though mROI changes require explicit synchronisation to ensure the correct spatial registration of the pixels captured by the frame grabber. Pixels are processed to measured source positions and the pixel space error computed. This is then converted to an error in arc seconds and written to a memory page in reflective memory on the Gemini synchrobus ring network.

The original RTC computed guide star position errors based on pulse counts from the APDs. In the NGS2 system the RTC takes the position errors as inputs, reading them from the reflective memory location that is written by the detector control process.

The NGS2 control system is based on a Intel architecture PC running an Linux operating system. A CameraLink PCIe frame grabber card and PCI reflective memory card support the physical interfaces to the detector system and RTC respectively.

\section{OPERATIONAL CONCEPTS}

NGS2 will require a limited number of changes in the observation planning and the observation execution tools, as well as operational software like the MYST real-time display, all of which are the responsibility of Gemini.

Changes to the OT (Observing Tool) include allowing for much fainter guide stars (and may reveal catalog shortcomings), removing the patrol area limitations, checking and flagging for asterism guide star magnitude difference, and updating the noise model of the Strehl estimator.

Change in the acquisition and operation observation procedures will exploit the new capability to image the entire $2^{\prime}$ field of view. Essentially, once pointed on the field, the operator requests NGS2 to read in full frame mode (3 sec overhead for mode switch) and checks the field and guide star positions with respect to the location of the guide box, where the guide stars were expected based on catalog positions. The operator has then the possibility to correct overall pointing, recenter individual guide stars, or even change guide star entirely if a more suitable one is available - either brighter or more strategically located.

As described in section 4.4, the fact that NGS2 offers interface hooks that essentially mimic the ones of the current NGSWFS minimises significantly the effort in upgrading the telescope-side tools. The NGS2 frame or 


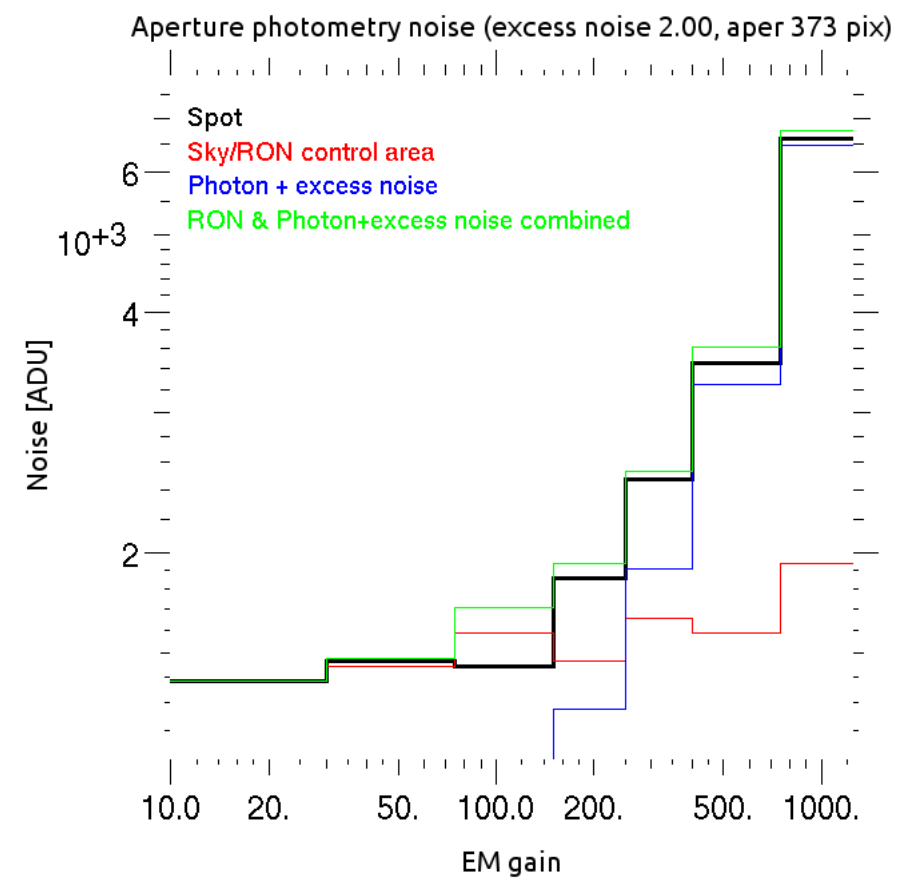

Figure 6. Noise as a function of EMCCD gain, or checking that the excess noise behaves as expected: In red, the sky/RON as measured in a control area, which is, as expected, relatively independent from the EMCCD Gain. In blue, the photon + excess noise as modeled using an excess noise $\mathrm{F}^{2}=2$. In green, the sum of the previous two contributors. In black, the measured noise.

subframe can be displayed $(>5 \mathrm{~Hz}$ ) using MYST -the GeMS high level real-time display- or with the NGS2 engineering GUI.

When an offset is requested, NGS2 updates the mROI positions following the TCS (Telescope Control System) directives. When working at constant mROI sizes, the HNü-512 reconfiguration is essentially seamless, with no noticeable overhead nor dropped frame. As shown in the performance section (6), we expect to use most commonly $4 \times 4$ or $6 \times 6$ pixel ROIs.

Because of differential atmospheric refraction, or science instrument-to-NGSWFS differential flexure, the TCS will change the guide window location. This is done at $20 \mathrm{~Hz}$ and will be followed by the NGS2 computer that will update the NGS reference positions and offset the ROIs when the reference position gets over a critical value. A 1 pixel padding is used during the initial configuration to allow some slack. Reconfiguration beyond this, if needed, is very fast and will not induce any skipped frame.

Initial system calibration during commissioning will include photometric and coordinate system calibrations, as well as establishing a distortion map of the NGS WFS focal plane. For the latter, we will use moderately dense astrometric fields, which should provide accurate references.

\subsection{NGS focus sensing with PWFS1}

As mentioned earlier, one drawback of the focal plane solution is that an alternative solution providing the slow focus information must be found to compensate for the sodium layer altitude variations. The proposed solution is to use an off-axis PWFS1 (Peripheral WFS 1) to monitor for focus drift and compensate by moving the LGS WFS focus. Gemini has been using a "LGS+P1" mode for a few years ${ }^{5}$ with success and proposes to extend this mode to focus sensing when operating with GeMS. 


\section{EXPECTED PERFORMANCE}

In this section, we report on detector tests done at ANU in 2015, as well as extensive Monte-Carlo simulations to assess performance and investigate various limitations.

\subsection{Detector tests}

The Nüvü HNü-512 was delivered in July 2015. Tests were carried out at the ANU on Sept 7-9, 2015, in presence and with the help of Gemini personnel. Tests show that the camera performs as expected, at a level consistent with the performance measured by Nüvü in the camera certificate of conformity (generated during the camera Factory Acceptance Tests), and specified in the quote for the camera, which was itself derived from the NGS2 camera specifications. Table 2 reports on the main Nüvü HNü-512 detector characteristics, as reported by the vendor in the certificate of conformity and checked at the ANU.

Table 2. Nüvü HNü-512 EMCCD specifications

$\begin{array}{ll}\text { Parameter } & \text { As built } \\ \text { Format } & 512 \times 512 \\ \text { EM gain } & \text { Up to } 5000 \\ \text { RON (20MHz H.Freq) } & \approx 300 / \text { EM_gain } \\ \text { CIC (20MHz H.Freq) } & 0.00058 \mathrm{e}^{-} / \text {pix } / \text { frame } \\ \text { Dark Current } & 0.00050 \mathrm{e}^{-} / \mathrm{pix} / \mathrm{s} \\ \text { Number of ROI } & \text { Up to } 8 \\ \text { Cosmetic defect } & \text { None (Science grade) } \\ \text { Effective full well (see text) } & 800 \mathrm{ke}^{-} / \text {EM_gain }\end{array}$

The CIC (Clock Induced Charge) is one of the lowest on the market, which is relevant for our application. Up to 8 ROIs can be programmed, of arbitrary size. For each ROI, the entire width of the CCD is read out; it is the receiving computer that deals with the $\mathrm{X}$ windowing. Reconfiguration of the detector from full frame to ROI, change in ROI size, takes 3 seconds. Simple moves of the ROI coordinates are immediate (from one frame to the next). The noise behaves as expected. The Clock Induced Charge is actually lower than expected and the excess noise is at the expected level.

Table 3. Nüvü HNü-512 read out modes

$\begin{array}{llll}\text { Mode } & \text { Horizontal frequency } & \text { Vertical Frequency } & \text { Note } \\ 9 & 1 \mathrm{MHz} & 1 \mathrm{MHz} & \text { conventional mode } \\ 16 & 20 \mathrm{MHz} & 2 \mathrm{MHz} & \text { Optimal for noise and CIC } \\ 17 & 20 \mathrm{MHz} & 3 \mathrm{MHz} & \text { Speed boost allows } 800 \mathrm{~Hz} \text { frame rate }\end{array}$

Table 3 lists the main modes that will be used for the camera. Mode 9 will be used during acquisition (Max rate $3.7 \mathrm{~Hz}$ ). Mode 16 and 17 will be used during regular mROI operation. These modes are referred to in table 4 that gives the maximum frame rate for various modes and ROI sizes. In Mode 16, the full-frame maximum frame rate is $62.6 \mathrm{~Hz}$. In mROI mode, the maximum frame rate depends on the number of ROIs, their size, and the Y coordinates of the last ROI (i.e. number of lines that have to be flushed before getting to the last ROI). In mode 16, for 3 ROIs of 8 pixels high, the maximum frame rate is given by $812-0.273 * N$, where $N$ is the the line number of the last ROI last read-out row. At worst $(N=511)$, in mode 16 , the frame rate is $681 \mathrm{~Hz}$ for 3 ROIs of 8 pixels high. The maximum frame rate is thus, assuming $6 \times 6 \mathrm{ROIs}$, at or above the required $800 \mathrm{~Hz}$.

In a final end-to-end test, a spot of FWHM a few pixels was formed on the HNü-512. Flux was reduced down to a few tens of photons per frame. A series of images was taken and analysed at various EM gain levels. Here we report about aperture photometry tests. Results are shown in figure 6 . The black line shows the actual measurements (rms of the aperture photometry signal versus sample number). The red line traces the noise in a 
Table 4. Measured maximum frame rate for various modes.

$\begin{array}{lll}\text { Mode } & \text { Max frame rate }[\mathrm{Hz}] & \text { Notes } \\ 9 & 3.69 & \text { Default acquisition mode }(9.7 \mathrm{e}-\mathrm{RON}) \\ 16 & 62.61 & \text { Full frame } \\ 16 & 821-0.273 \times \mathrm{N} & \text { mROI } 3 \times 8 \text { pixel bands, } \mathrm{N} \text { is line \# of top ROI top row } \\ 16 & 712 & \text { mROI } 3 \times 8 \text { pixel bands, } \mathrm{N}=400 \\ 16 & 681 & \text { mROI } 3 \times 8 \text { pixel bands, } \mathrm{N}=511 \\ 17 & 821 & \text { mROI } 3 \times 8 \text { pixel bands, } \mathrm{N}=400 \\ 16 & 815 & \text { mROI } 3 \times 6 \text { pixel bands, } \mathrm{N}=400\end{array}$

control area close to the spot but with no light from the spot itself; the blue line is a plot of the photon noise and excess noise, as modeled from theory with an excess noise of 2.0, knowing how many photons there is (measured with the ADU gain, itself calibrated with a photon transfer curve at EM gain of unity); Finally, the green line is the background noise, photon noise and excess noise (red+blue lines). This green curve fits the measurements very well, which means that our description of the excess noise is modeling the reality well.

In summary, both in photometry and astrometry, the camera behaves as expected from a standard EMCCD model, which validates the simulation results presented below.

\subsection{Simulations}

A number of simulations have been carried out, to investigate the effect of read-out noise, excess noise, ROI size, etc, on performance. Simulations were done with yao. ${ }^{6}$ We investigated only the effect of TT guiding on a single on-axis star. This is not only necessary - as otherwise the parameter space includes the asterism geometry, which would increase the number of parameters enormously - but is also justified because the asterism geometry is actually not relevant to what we want to simulate, which is the performance of a TT WFS in terms of noise. Noting that the high order loop performance is independent of the TT and plate scale loop, we generated a batch of high order corrected images in a number of conditions, and used these images to feed the modeled NGSWFS (650nm effective wavelength). Noise (photon, excess and read-out noise consistent with the detector characteristics as presented in the previous section), TT loop dynamic, wind-shake (400mas $\mathrm{PV}$ at $0.5 \mathrm{~Hz})$ is then applied in a close loop simulation, and combination of the high order kernel and TT residual is then used to produce the final image at $2.2 \mu \mathrm{m}$.

On top of pure performance versus magnitude simulations in terms of residual Tip-Tilt, we also investigated the following:

- The impact of the limited dynamical range of the EMCCD on the SNR of faint GS when a bright GS is in the asterism,

- The presence of a small amount of aberration in the image (e.g. astigmatism),

- The effect of not being exactly centred on one pixel,

An executive summary of the simulation results is encapsulated by the following statements:

- The simulation shows that there is a significant margin to reach the top level science requirement (less than 35mas rms residual TT at $\mathrm{R}=17$ ).

- The EMCCD limited dynamical range does not affect in any significant way the performance of the system. It has marginal impact that can be mitigated by simple changes in the observation preparation tool.

- Additional uncompensated static astigmatism in the system at the level of $250 \mathrm{~nm}$ will reduce sensitivity by 0.05 magnitude under median seeing and 0.12 magnitude under 20 percentile seeing.

- The provided sampling of $0.235^{\prime \prime} /$ pixel is not inducing any detectable non-linear effect when the star is not centred at the apex of four pixels. 
Figure 7 shows the performance simulation results. The top two figures show the RMS Tip-Tilt residuals versus magnitude. The bottom two figures show the final $2.2 \mu \mathrm{m}$ image FWHM -combination of the RMS TT residual and the high order image- versus magnitude. The left two plots are for various seeing conditions as indicated on the plots (ROI 6x6). The right two plots are for various ROI sizes (seeing $13.4 \mathrm{~cm}$ ).

The top plots of figure 7 show that the degradation with seeing is relatively smooth (left), but also shows that the dependency on the ROI size is quite strong: for faint guide stars $(\mathrm{R} \geq 18)$, a $4 \times 4$ ROI does twice better
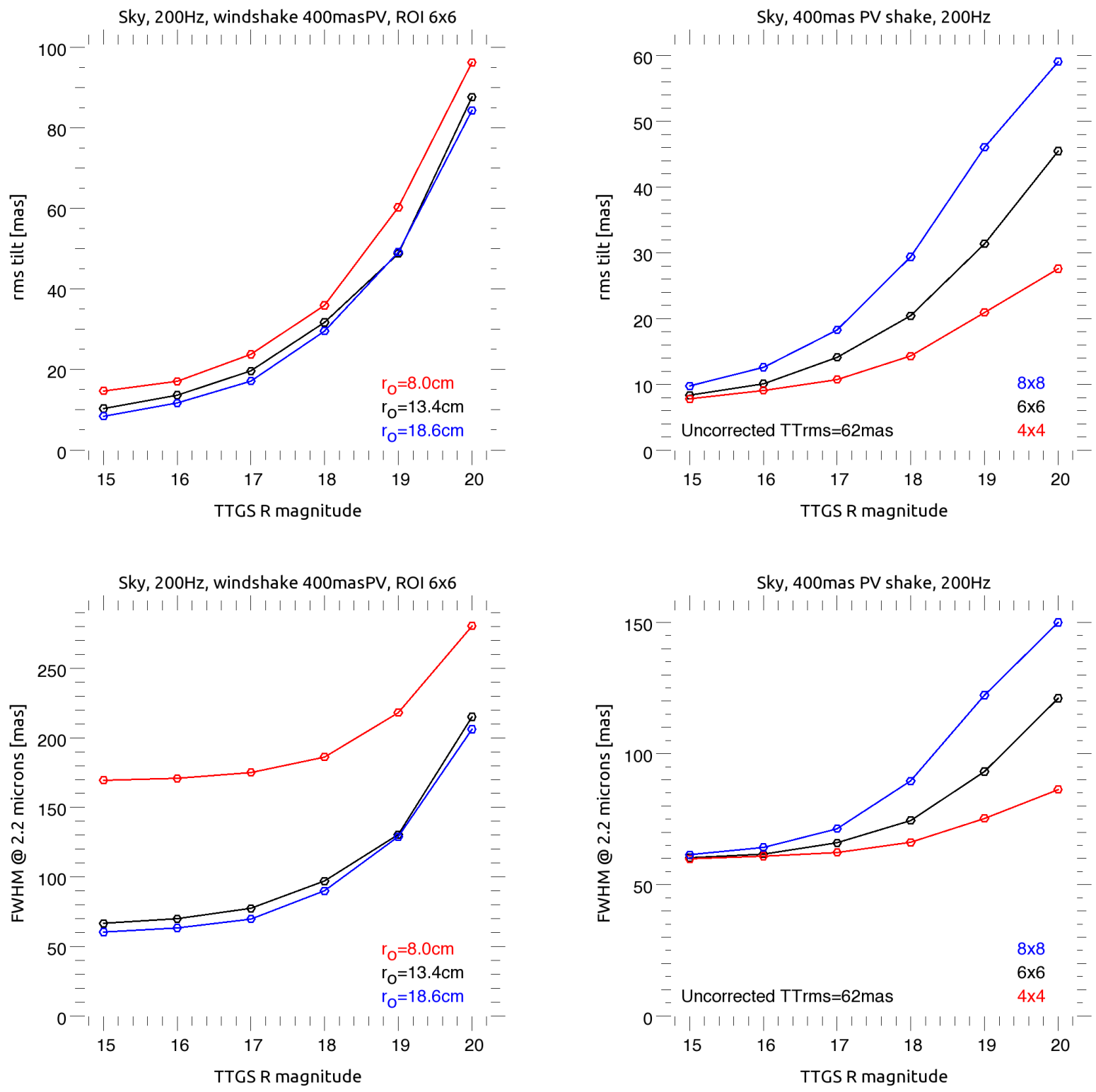

Figure 7. NGS2 Simulations. Top: RMS tilt (one direction) versus guide star R magnitude for various $r_{0}(500 \mathrm{~nm})$ conditions and a 6x6 ROI (left), and for various ROI sizes and r0 $=13.4 \mathrm{~cm}$ (right). Bottom: Overall FWHM of the image at $2.2 \mu \mathrm{m}$ versus the guide star magnitude. Left: ROI $6 \times 6$, various $r_{0}(500 \mathrm{~nm})$ as indicated in the figure. Right: Same $r_{0}(500 \mathrm{~nm})=13.4 \mathrm{~cm}$, but various ROI size, as indicated in the figure. Note that the effect of the high order correction is taken into account, thus the FWHM in the bottom left figure is dominated by high order error and the FWHM of the short exposure images, explaining the large FWHM even when using a bright NGS. 

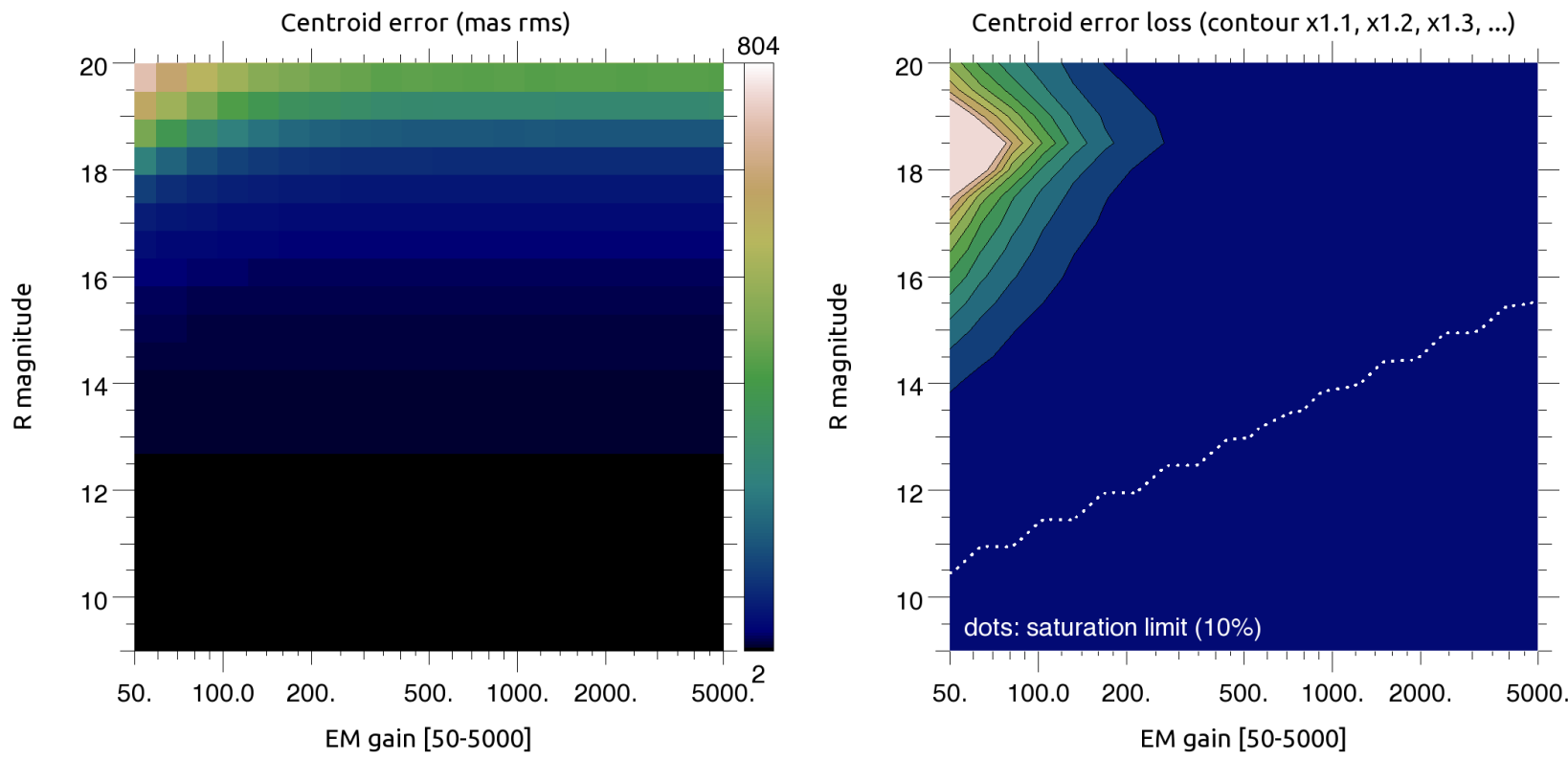

Figure 8. Left: Centroid error, for magnitudes $\mathrm{R}=9$ to 20 and EM gains from 50 to 5000 . Right: Centroid error loss for a $6 \mathrm{x} 6$ window, i.e. for each magnitude, factor by which the centroid error is degraded at given EM gain, compared to the centroid error for the optimal EM gain. The contours are spaced by 0.1, i.e. 1.1, 1.2, 1.3, etc, with a maximum of 2.0 (light pink colour). The dotted "line" indicates the limit at which $10 \%$ of the images saturate the EM register full well.

than a $8 \times 8$ ROI. Even using an EMCCD, the number of pixels, or subaperture field of view, is very relevant in term of performance; it is always better to use the smallest possible window to get the lowest noise. This is of course balanced by the need to get enough field of view to avoid vignetting and/or losing the star altogether. $6 \times 6$ is not optimal in terms of performance, but seems like a safe compromise between field of view and noise, and it has been our baseline throughout the simulation work.

The bottom plots of figure 7 (TT residual + high order image) are really the end-to-end performance metric of interest. The floor at $\approx 65$ mas is the core of the high order corrected PSF in good performance conditions of the high order loop. The overall performance degrades smoothly with fainter Tip-Tilt guide stars. The left plot shows that the performance breaks down in bad seeing conditions, but this is more a consequence of the high order loop not being able to cope than the TT correction loop, as shown by the large FWHM increase for bright TT guide star. It also shows that for median seeing or better (black and blue curves), a FWHM $\leq 100 \mathrm{mas}$ is reached for NGS magnitude $\mathrm{R} \leq 18$, which is good news for the science to come.

\subsubsection{Limited dynamic range}

EMCCDs are known for their sensitivity, but not for their large dynamic range. NGS2 will be dealing with up to three guide stars, which will likely be of different brightness. The maximum EM gain will be set by the need to not saturate the EM registers on the brightest star. Consequently, other, fainter guide stars in the same asterism may not be observed with the optimal EM gain and SNR may suffer.

To that effect we simulated a large set of centred images, and applied the EMCCD properties, in terms of amplification and noise. The results are presented in figure 8. We ran the simulation for a set of magnitudes (from 9 to 20) and EM gains (from 50 to 5000). For each magnitude, a set of points, centroid errors versus EM gain was obtained and is shown in the left hand panel of figure 8. The right hand panel presents the centroid error loss for each magnitude: it is the centroid error divided by the minimum error for each magnitude. In effect, for each magnitude, it is a loss, versus the EM gain, with respect to the best EM gain choice. A value of the centroid error loss of 1.5 means that the error is 1.5 larger than the one that could have been obtained had the EM gain been chosen properly. Figure 8 (right) shows the re-assembled array, centroid error loss for 
the given star magnitudes and EM gain. The dark blue plateau is for no loss, i.e. a centroid error loss equal to 1. Each subsequent contour is separated by 0.1 , i.e. 1.1, 1.2, 1.3 etc, up to 2.0 for the contour level coloured in light pink.

Several things can be noted from figure 8:

- The smaller centroid error (or here, centroid error loss) is always for the highest EM gain. This is normal, as, in our modeling, the statistics of the EM gain register does not depend on the EM gain.

- For bright stars $(\mathrm{R} \leq 14)$, there is no penalty using a small EM gain (uniformly blue irrespective of the EM gain). For fainter stars, using smaller EM gains result in larger centroid errors, because the RON is not insignificant. This effect is more and more pronounced for fainter stars, and then becomes less pronounced for $\mathrm{R}>18.5$. This is because what we show here is a relative loss, and the absolute centroid error obtained on these very faint stars is very large, thus reducing the impact of the additional RON (in other words, not much is lost by going from bad to worse).

- To determine how much, if any, additional centroid error is incurred by the combination of EM gain and brightness contrast in the asterism, one can read figure 8 as follow:

1. Take the magnitude of the brightest star in the asterism.

2. What is the largest EM gain (emg0) at which this star can operate without saturating?

3. Look for the centroid error loss on the fainter asterism stars, at this EM gain emg0.

For instance, for an asterism with two stars of $\mathrm{R}=(13,17)$ : One can operate safely on the $\mathrm{R}=13$ with an EM gain of 300. At this gain, for the $\mathrm{R}=17$ star, the centroid error loss is smaller than 1.1. For a $\mathrm{R}=(12,18)$ asterism (an extreme case), the system will have to be operated at an EM gain of 140, which leads to a loss of approximately $30 \%$ on the fainter guide star centroid error.

- Another conclusion of this figure is that the use of EM gains larger than $\approx 300$ is unnecessary. At 300, the centroid error loss is negligible over the whole magnitude range.

- Finally, note that even at low EM gains (50), the brightest star that will not saturate is $\mathrm{R}=10.5$.

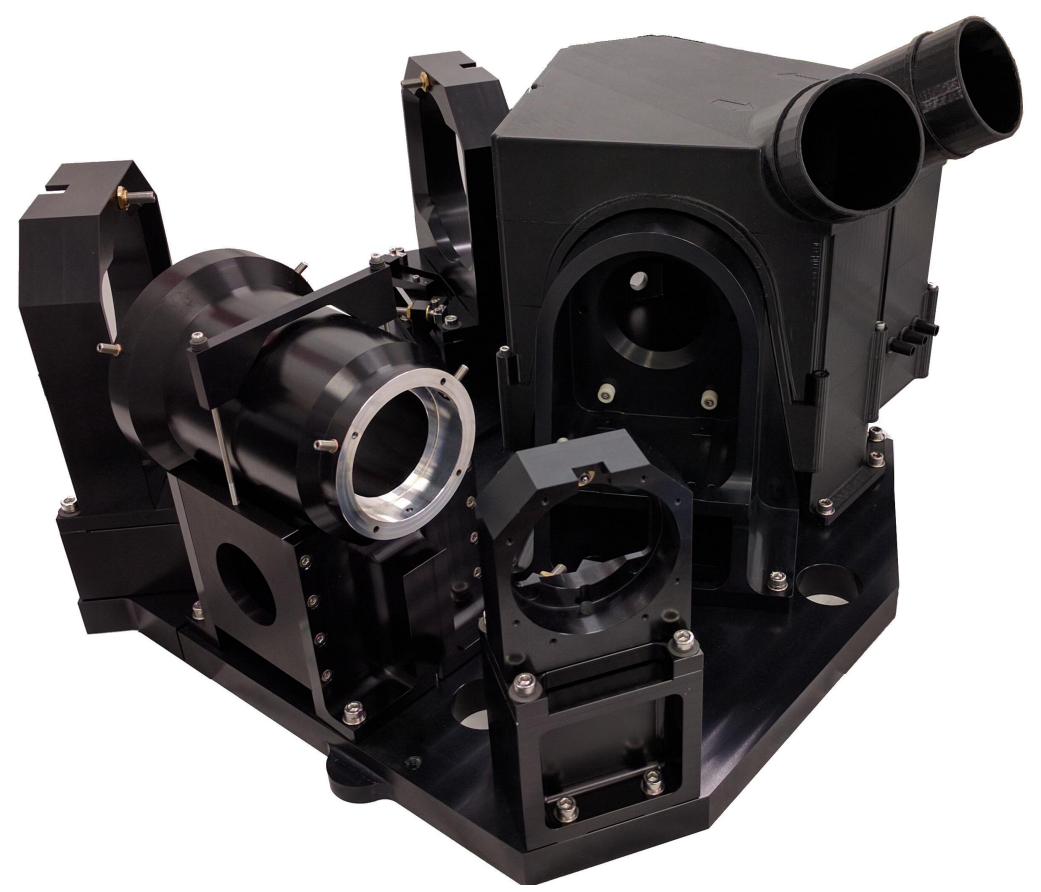

Figure 9. Hardware in the lab as it stands in May 2016. The lenses have been received but are not yet mounted. The objective lens is not yet in place. The big black box in the upper right is the thermal enclosure of the HNü-512 EMCCD. 
The overall conclusion is that, most of the time, the limitation induced by the limited EM registers well depth is not going to affect performance, with the exception of extreme cases of asterism contrast when the brightest guide star has a $\mathrm{R}<12.5$ and others have $\mathrm{R}>16-17$.

\section{CONCLUSION}

NGS2 is an upgrade for the Tip-Tilt WFS of GeMS. It will vastly improve performance on faint guide stars, and push the limiting magnitude from the current $R=15$ to $R \geq 18$. It will also streamline acquisition and solve the issues associated with geometric focal plane distortions, which currently induce dramatic limitations for offsetting and image stacking.

Figure 9 shows NGS2 in the ANU lab on May 2016. In the current plan, NGS2 should go through acceptance tests at the ANU before the end of 2016, and be integrated in GeMS and commissioned at Gemini in the course of 2017 .

\section{ACKNOWLEDGMENTS}

This research was supported under Australian Research Council's Linkage Infrastructure Equipment and Facilities funding scheme (project number LE140100013). We acknowledge financial support from the Australian National University, the Swinburne University of Technology, the Australian Astronomical Observatory and the Gemini observatory.

The Gemini Observatory is operated by the Association of Universities for Research in Astronomy, Inc., under a cooperative agreement with the NSF on behalf of the Gemini partnership: the National Science Foundation (United States), the National Research Council (Canada), CONICYT (Chile), the Australian Research Council (Australia), Ministério da Ciência, Tecnologia e Inovação (Brazil) and Ministerio de Ciencia, Tecnología e Innovación Productiva (Argentina).

\section{REFERENCES}

[1] Rigaut, F., Neichel, B., Boccas, M., d'Orgeville, C., Vidal, F., van Dam, M. A., Arriagada, G., Fesquet, V., Galvez, R. L., Gausachs, G., et al., "Gemini multiconjugate adaptive optics system review-i. design, trade-offs and integration," Monthly Notices of the Royal Astronomical Society 437, 2361-2375 (2014).

[2] Neichel, B., Rigaut, F., Vidal, F., van Dam, M. A., Garrel, V., Carrasco, E. R., Pessev, P., Winge, C., Boccas, M., d'Orgeville, C., et al., "Gemini multiconjugate adaptive optics system review-ii. commissioning, operation and overall performance," Monthly Notices of the Royal Astronomical Society 440(2), 1002-1019 (2014).

[3] Djazovski, O., Daigle, O., Laurin, D., Bedirian, M., Ducharme, M.-E., Artigau, É., and Doyon, R., "Electronmultiplying ccds for future space instruments," in [Photonics North 2013], 89150Q-89150Q, International Society for Optics and Photonics (2013).

[4] Rigaut, F., Neichel, B., Boccas, M., d'Orgeville, C., Arriagada, G., Fesquet, V., Diggs, S. J., Marchant, C., Gausach, G., Rambold, W. N., et al., "Gems: first on-sky results," in [Proc. SPIE], 84470I-84470I, International Society for Optics and Photonics (2012).

[5] "Lgs + p1 'super seeing' mode." https://www.gemini.edu/pio/?q=node/11782. Accessed: 2016-Jun-26.

[6] Rigaut, F. and van Dam, M., "Simulating astronomical adaptive optics systems using yao," in [AO4ELT3], Esposito, S. and Fini, L., eds. (2013). 